\title{
An Approach to Psychological Assessment of Chronic Pain Patient Evaluated for ImPLantable DeVICES
}

\author{
Saba Javed, MD, and Billy K Huh, MD, PhD
}

Chronic pain is a multidimensional field in which a patient's physical, environmental, and psychological state must be fully understood to further assess their problem. These variables form a complex interaction with each other and play a critical role in the treatment of chronic pain. The clinical interview is the cornerstone of the psychological evaluation. An important purpose of the interview is to identify any psychiatric conditions that might exacerbate pain or complicate treatment, such as psychosis, substance dependence, or a personality disorder.

The patient was seen in the chronic pain clinic and assessed by the pain clinical psychologist.

We present the case of a patient who presented to the chronic pain clinic and underwent evaluation for spinal cord stimulator trial placement. As part of this evaluation, the patient underwent psychological assessment by the pain clinical psychologist; we discuss the approach to psychological assessment using observational narrative.

Psychological evaluation can help guide a physician's treatment plan to better meet the patient's needs. Psychological assessment prior to interventional management should be used as a tool to give the patient the best chance for success.

Key words: Psychological assessment, psychological evaluation, spinal cord stimulation, intrathecal pump
Chronic pain is a multidimensional field in which a patient's physical, environmental, and psychological state must be fully understood to further assess their problem. These variables form a complex interaction with each other and play a critical role in the treatment of chronic pain. Instruments like the Multidimensional Pain Inventory (MPI), which consists of 56 items to assess the psychosocial, cognitive, and behavior aspects of pain, might help gauge a patient's response to a particular treatment $(1,2)$. For instance, it has been shown that psychiatric risk factors such as depression, stress, psychological vulnerability, and duration of disability are associated with chronic

From : MD Anderson Cancer Center, Houston Texas

Author for correspondence: Saba Javed, MD

Address: MD Anderson Cancer Center, 1515 Holcombe Blvd., Houston, Texas 77030

E-mail: sabajaved23@gmail.com postsurgical pain (3). Another study found that a significant number of patients who failed spinal cord stimulator therapy might have had improved treatment outcomes if only they had had a preoperative psychosocial assessment before and after their therapy (4). These studies presented above cannot further emphasize the importance of psychological testing in patients who undergo invasive treatments for their chronic pain.

The clinical interview is the cornerstone of the psychological evaluation. An important purpose of the interview is to identify any psychiatric conditions that might exacerbate pain or complicate treatment, such as psychosis, substance dependence, or a personality disorder. The Centers for Medicare and Medicaid Services (CMS) in the United States mandates the use of preimplantation psychological screening for spinal cord stimulation (SCS) in an effort to improve outcomes. Nelson et al proposed a list of red flags including suicidal tendency, alcohol 
or drug dependency, unresolved compensation/legal issues, severe untreated depression, and other factors (5). Moreover, inquiring about pain behaviors and functional status can provide important information about the person's overall pain experience, coping skills, as well as the extent of pain-related disability.

The objective of this article is to present a case report and discuss an approach to psychological assessment as the patient is evaluated for possible spinal cord stimulator (SCS) placement.

\section{CASE REPORT}

Patient $A$ is a 58-year-old morbidly obese woman with a remote history of bipolar disorder and major depressive disorder, fibromyalgia, and lymphoma status post chemotherapy with no current evidence of disease; she has been followed in the pain clinic for chronic lower back pain due to lumbar spondylosis. The patient's pain was located in her mid-lower back and she described the pain as dull, achy, and constant with episodes of sharp, radicular pain down her feet. Along with the above, the patient endorsed weakness in her legs and paresthesia. Her current pain regimen consisted of pregabalin, methocarbamol, and tapentadol. The patient had tried numerous pain medications in the past including gabapentin, tramadol, hydrocodone, oxycodone, hydromorphone, and methadone; however, she developed side effects from these pain medicines. In addition to medications, the patient had undergone a number of interventional pain procedures including lumbar epidural steroidal injection, medial branch block, and radiofrequency ablation. These procedures provided her with only fractional and short-term relief. During her most recent follow-up at the pain clinic, the patient stated that her quality of life was extremely poor due to the pain and she was interested in any procedure that could help her. We discussed a trial of SCS and the patient seemed very much interested. Identified as a possible candidate for SCS, the patient was sent to a clinical psychologist to have a psychological evaluation.

During the psychological assessment interview, the patient stated that she had a difficult upbringing by her parents wherein they were both physically and emotionally abusive toward her. During her teens and early twenties, she suffered from bipolar disorder and major depressive disorder and was followed by a psychiatrist. At 16 years of age, she attempted to commit suicide by overdosing on prescription medication. The patient also stated that she had numerous psychiatric admissions for when she had thoughts of hurting herself. The patient was currently not seeing a psychiatrist and was taking lorazepam for anxiety and zolpidem for bedtime as her only psychiatric medications. In addition to the above psychiatric history, the patient said that her mood and pain affected her functional status. She noted that she was spending upwards of 20 hours a day in bed due to pain. She sometimes would forgo bathing or showering because of the excruciating pain. The patient shared an apartment with a male companion. For a living, she ran her own business of selling paintings, and while she was in bed upwards of 20 hours a day, she would blog on her computer for at least a portion of that time. The patient complained that she did not sleep well at night and would wake up numerous times due to pain. She endorsed that she was not happy with her current lifestyle and the person she had become and would like to make changes in her routine.

\section{DISCUSSION}

An important objective of doing a psychological assessment in a patient being evaluated for implants (for example SCS, dorsal root ganglion, or intrathecal pump) is to identify any psychiatric conditions, functional status, history of substance abuse and/ or suicide, hygiene, and motivation that might exacerbate pain or complicate treatment. Psychological assessment before surgery has been an important factor in determining better outcomes. It has become standard practice by insurance companies that in order to approve a patient for SCS, dorsal root ganglion, or intrathecal pump placement, the patient must undergo a psychological assessment by a clinical psychologist. This is usually done in the form of a structured clinical interview in order to identify any psychiatric conditions that might exacerbate pain or complicate treatment, such as psychosis, substance dependence, or a personality disorder. A standard psychological interview inquires about the patient's pain history, how their pain impacts their quality of life, personal medical history and psychiatric history, family history, and medications. 


\section{Psychiatric History and Mood Disorder}

As discussed above, patient A had an extensive history of mood disorder starting in childhood. Patient $A$ also had numerous suicidal attempts by prescription medication overdose and multiple psychiatric hospitalizations. Looking at the literature, mood spectrum disorders worsen the perception of pain, increasing both its intensity and various components of pain. A large number of studies have investigated the connection between depression and chronic pain, an association supported by the fact that they share some activation/dysregulation of brain structures (6). In particular, the anterior cingulate cortex (ACC), amygdala, and prefrontal cortex encode emotional and cognitive aspects of chronic pain, and are also involved in the experience of emotion and the processing involved in emotional memory formation (7).

Moreover, individuals with chronic pain are at increased risk for suicidal thoughts and behavior. Comorbid psychiatric disorders like depression, prevalent in chronic pain, also increase the risk for suicidality. There are a number of factors that increase the risk for an individual to attempt suicide in the general population such as past suicide attempts, family history of suicide, lack of social support, and access to means of suicide such as weapons or medication. For chronic pain patients, additional risk factors include the presence of comorbid depression, high pain intensity and duration, the presence of sleep-onset insomnia, high catastrophizing, and feelings of hopelessness (8). Hence, extreme caution must be taken when considering individuals with an extensive prior psychiatric history.

\section{Substance Use and Opioid Medication Misuse}

Patient A denied any history of past or current substance use or alcohol use. However, she had endorsed history of opioid medication misuse, as stated above. On a few instances, she overdosed on prescription opioids in a suicidal attempt. The prevalence of alcohol/substance use and prescription opioid misuse in persons with chronic pain is significant; thus, a comprehensive evaluation should include screening for alcohol, substance, and prescription opioid use. Assessment of alcohol and substance abuse is important in the context of treatment for chronic pain since individuals with substance use disorder are at an increased risk for opioid misuse (9).
Precise rates of opioid medication misuse have been difficult to establish, but estimates of the prevalence of opioid dependence in pain clinic settings could be as high as $14 \%(10)$. Needless to say, patients with prior history of substance use/opioid misuse should be identified and evaluated during psychological assessment; the decision to proceed with implant should be taken with extreme caution and only after the patient exhibits sufficient knowledge, insight, and compliance with the plan.

\section{Pain-related Disability and Behavior}

It is suggested that pain can cause disability and depression, as pain can limit physical movements and thus cause disability. In turn, disability reduces movements, causing pain, turning into a vicious cycle. In this case report, patient $A$ described herself as physically disabled as the pain had severely limited her activities of daily living and she was confined to her bed for over 20 hours each day. Disability prevents people from doing activities they used to engage in, such as housework, sports, social interaction, sleep routine, etc., allowing depressive symptoms to appear; likewise, depressed patients tend to get more isolated and lose motivation to engage in any kind of activity (11).

Sleep disturbances are common in chronic pain patients and are known to be risk factors for both the development and exacerbation of pain. In this case report, when patient $A$ was asked about her functional status, she admitted to a bed-ridden lifestyle, staying in bed for upwards of 20 hours and not being physically active for the past 3 years due to pain. Furthermore, she stated that even though she was in bed, her sleep pattern was incredibly poor, as she would wake up about every hour. In a recent prospective study by Emery et al, the authors found that chronic pain patients exhibited more dysfunctional beliefs about sleep, poorer sleep hygiene practices, and greater pre-sleep arousal (12). Moreover, it is widely accepted that sleep, functional status, and overall outlook have an impact on recovery and postsurgical and long-term outcomes. As a case in point, in a recent article, patients over the age of 60 years who were admitted for arterial vascular surgery had longer hospital stays and adverse postoperative outcomes when their functional status and cognition were impaired (13). Since placement of a SCS can have 
negative consequences, patient A's lifestyle alone could have put her at risk for worse postoperative outcomes and failed treatment.

\section{Personal Hygiene}

It has been shown that lack of proper hygiene can increase the risk of surgical site infections; in fact, the Centers for Disease Control and Prevention (CDC) recommends that patients take shower or full baths with antimicrobial soap at least the night before surgery to reduce the risk of surgical site infection (14). Patient A stated during the psychological assessment that she would sometimes go days without showering or bathing due to the pain. She also noted that she would get 'sores' on her skin that would open up and drain from time to time, likely infectious pustules. Due to the patient's inability to maintain proper hygiene and self-care, and her being prone to repetitive skin infections, these factors potentially put her at even greater risk for postoperative complications.

\section{Compliance}

During the psychological assessment, patient $A$ was asked about compliance, both in terms of taking medications as scheduled and maintaining follow-up appointments. She was forthright about admitting to not taking her scheduled medications at appropriate times and then having to play catch-up. Furthermore, the patient stated that she had to miss appointments consistently due to long travel time and financial burden. During the psychological assessment, it was clear that her depressed state and psychiatric history had hindered her ability to find a proper pain management regimen. A study has shown that depressed patients are 3 times more likely to be noncompliant with medical treatment recommendations when compared to their nondepressed counterparts (15). The use of a SCS may be a temporary measure to help improve the patient's pain, but their lack of compliance with other medical regimens may make them more likely to fail treatment in the future.

\section{Financial Burden}

Patients with lack of financial support and huge financial burden are more likely to be noncompliant with therapy or medications (15). In patient A's case, she stated that her health care cost was a huge stressor in her life. Currently on Medicare and disability insurance, the patient was tailoring her medical care and oftentimes missing appointments simply because of her co-pays and out-of-pocket expenses. It comes as no surprise that financial stressors have a major impact on health outcomes (16). While a particular medication or the newest cutting-edge procedure can be the ideal solution to a particular chief complaint, every patient should be looked at individually and as a whole. While the patient can receive a SCS, if she is not able to maintain her follow-up appointments due to mounting co-pays or long-distance travel to see a physician, then a less invasive approach through medication optimization should be taken first.

\section{CONCLUSION}

Psychological assessment to screen candidates for interventional procedures has been an important tool to help determine the success of a treatment. These assessments not only help recognize a patient's chronic problem but can also help identify any red flags in areas such as psychiatric history/mood disorder, substance use/opioid medication misuse, pain-related disability/behavior, personal hygiene, compliance, and financial burden that should be addressed and optimized prior to surgery. These factors can help decide whether a procedure is even the right step for the individual or if alternatives should be sought. Looking into the psychosocial aspect of a person's life can help guide a physician's treatment plan to better suit the patient. Psychological assessment prior to interventional management should be used as a tool to give the patient the best chance for success. 


\section{REFERENCES}

1. Gatchel RJ, Noe CE, Pulliam C, Robbins H, Deschner M, Gajraj NM, Vakharia AS . A preliminary study of multidimensional pain inventory profile differences in predicting treatment outcome in a heterogeneous cohort of patients with chronic pain. Clin J Pain 2002; 18:139-143.

2. Junghaenel DU, Keefe FJ, Broderick JE. Multi-modal examination of psychological and interpersonal distinctions among MPI coping clusters: A preliminary study. J Pain 2010; 11:87-96.

3. Weinrib AZ, Azam MA, Birnie KA, Burns LC, Clarke H, Katz J. The psychology of chronic post-surgical pain: New frontiers in risk factor identification, prevention and management. $\mathrm{Br}$ J Pain 2017; 11:169-177.

4. Beltrutti D, Lamberto A, Barolat G, Bruehl SP, Doleys D, Krames E, Meglio M, North R, Olson K, Reig E, Simpson B, Turk D, Aronoff B, Turk D, Aronoff G, Melzack R. The psychological assessment of candidates for spinal cord stimulation for chronic pain management. Pain Pract 2004; 4:204-221.

5. Nelson DV, Novy DM. Psychological characteristics of reflex sympathetic dystrophy versus myofascial pain syndromes. Reg Anesth 1996; 21:202-208.

6. Baliki MN, Petre B, Torbey S, Harrmann KM, Huang L, Schnitzer TJ, Fields HL, Apkarian AV. Corticostriatal functional connectivity predicts transition to chronic back pain. Nat Neurosci 2012; 15:1117-1119.

7. Doan L, Manders T, Wang J. Neuroplasticity underlying the comorbidity of pain and depression. Neural Plast 2015; 2015:504691.

8. Tang NK, Crane C. Suicidality in chronic pain: A review of the prevalence, risk factors and psychological links. Psychol Med $2006 ; 36: 575-586$
9. Sehgal N, Manchikanti L, Smith HS. Prescription opioid abuse in chronic pain: A review of opioid abuse predictors and strategies to curb opioid abuse. Pain Physician 2012; 15:ES67-ES92.

10. Hojsted J, Nielsen PR, Guldstrand SK, Frich L, Sjogren P. Classification and identification of opioid addiction in chronic pain patients. Eur J Pain 2010; 14:1014-1020.

11. Garbi Mde O, Hortense P, Gomez RR, da Silva Tde C, Castanho AC, Sousa FA. Pain intensity, disability and depression in individuals with chronic back pain. Rev Lat Am Enfermagem 2014; 22:569-575.

12. Emery PC, Wilson KG, Kowal J. Major depressive disorder and sleep disturbance in patients with chronic pain. Pain Res Manag 2014; 19:35-41.

13. Partridge JS, Fuller M, Harari D, Taylor PR, Martin FC, Dhesi JK Frailty and poor functional status are common in arterial vascular surgical patients and affect postoperative outcomes. Int J Surg 2015; 18:57-63.

14. Segreti J, Parvizi J, Berbari E, Ricks P, Berrios-Torres SI. Introduction to the Centers for Disease Control and Prevention and Healthcare Infection Control Practices Advisory Committee Guideline for Prevention of Surgical Site Infection: Prosthetic Joint Arthroplasty Section. Surg Infect (Larchmt) 2017; 18:394400.

15. DiMatteo MR, Lepper HS, Croghan TW. Depression is a risk factor for noncompliance with medical treatment: Meta-analysis of the effects of anxiety and depression on patient adherence. Arch Intern Med 2000; 160:2101-2107.

16. Kahn JR, Pearlin LI. Financial strain over the life course and health among older adults. ] Health Soc Behav 2006; 47:17-31. 
